\title{
CAPTURE AND THREE DIMENSIONAL PROJECTION OF NEW SOUTH WALES STRATA PLANS IN LANDXML FORMAT
}

\author{
B. Harding, A. Foreman \\ Spatial Services, Department of Finance, Services and Innovation \\ brad.harding@finance.nsw.gov.au, ashleigh.foreman@finance.nsw.gov.au
}

KEY WORDS: Strata, 3D cadastre, New South Wales, Land administration, LandXML, ArcMap, ePlan

\begin{abstract}
New South Wales is embarking on a major reform program named Cadastre NSW. This reform aims to move to a single source of truth for the digital representation of cadastre. The current lack of a single source cadastre has hindered users from government and industry due to duplication of effort and misalignment between databases from different sources. For this reform to be successful, there are some challenges that need to be addressed.

"Cadastre 2034 - Powering Land \& Real Property" (2015) published by the Intergovernmental Committee on Surveying and Mapping (ICSM) identifies that current cadastres do not represent real property in three dimensions. In future vertical living lifestyles will create complex property scenarios that the Digital Cadastral Database (DCDB) will need to contend with. While the NSW DCDB currently holds over 3 million lots and 5 million features, one of its limitations is that it does not indicate land ownership above or below the ground surface.

NSW Spatial Services is currently capturing survey plans into LandXML format. To prepare for the future, research is being undertaken to also capture multi-level Strata Plans through a modified recipe. During this research, multiple Strata Plans representing a range of ages and development types have been investigated and converted to LandXML. Since it is difficult to visualise the plans in a two dimensional format, quality control purposes require a method to display these plans in three dimensions. Overall investigations have provided Spatial Services with enough information to confirm that the capture and display of Strata Plans in the LandXML format is possible.
\end{abstract}

\section{INTRODUCTION}

Spatial Services, a part of the Department of Finance, Services and Innovation is currently completing a project to back capture all existing surveyed plans into LandXML format. Land and Property Information NSW is developing a digital plan processing system for lodgement of plans in LandXML. This conforms to the national standard for digital lodgement set forth by ICSM (Land and Property Information, 2017). The back captured plans are intended to complete a set of digital plans for the entire state of NSW. At present, only deposited plans (i.e. survey plans of land subdivision) have been captured. To attain a complete set of all plans, a number of different plan types will eventually need to be converted, such as; Strata/Stratum Plans, Community Plans, Crown Plans, and others. This paper concerns itself with the capture of Strata Plans (SP's), as it is envisaged that successfully back capturing these will enable and guide the successful capture of other types. Strata capture may present a variety of scenarios and unique challenges compared to other plan types. Additionally, the capture of pre-existing strata plans should help to inform best practices for digital lodgement of new strata plans in future. Collected plans in LandXML format are intended to improve the accuracy and completeness of the DCDB.

A significant amount of high density development within NSW means that capturing a 3D cadastre is essential for the future (Hornery, 2015). A problem is that the current DCDB only shows land ownership at ground level, with no indication of what is below or above the surface. It is here that a captu red dataset of LandXML plans integrated into the DCDB could be beneficial.

The version of the NSW LandXML Recipe being used is V8.0.2 (Feb 2017). Land and Property Information have proposed changes to the version of the recipe to enable strata capture. At the time of writing these changes are still in discussion, however elements of the proposed changes have been used for the trial capture here. The following sections outline the method used for back 
capture operators to capture a Strata property in such a manner. The method is intended to be a proof of concept that will perhaps become more realised and refined over time.

As stated, specifications for implementation and the software to support it are still being finalised, however the initial test capture of some plans aims to assess the validity of strata capture for users as well as the New South Wales government as a whole. Stakeholder benefits from 3D strata plan capture are to be validated; the trial has helped identify issues in capture and highlighted some unexpected results. Some of these are presented later in this paper.

Through the conversion process, it was discovered that when a strata plan had been captured and converted to LandXML format, it was difficult to visually examine the file for the identification of errors. Projection in two dimensions was troublesome as well, as different floor levels were still represented on the same flat plane. Moreover, the projection of a plan into 3D presents a number of proposed benefits for potential users of the data within the community. The method used to display the plans in $3 \mathrm{D}$ as well as some of the potential for such plans is discussed.

The paper is structured as follows; firstly, some relevant studies are mentioned, followed by benefits and a discussion on some of the proposed changes to the LandXML recipe. The next sections detail the process undertaken to arrive at a basic 3D model before looking at issues, conclusions and possible future directions.

\section{OTHER RELEVANT STUDIES}

Outside of New South Wales, work in the fields of digital capture of strata properties and 3D representation has been performed by a number of private parties and government agencies. Of note and of inspiration to the studies presented here in particular are the works of Shojaei, Olfat,Rajabifard, Darvill and Briffa (2016) whose paper "Assessment of the Australian digital cadastre protocol in terms of supporting 3D building subdivisions" informed aspects of the 3D data modelling methods used.

Further work performed by the University of Melbourne shows that $3 \mathrm{D}$ cadastre research is well under way. The older article "3D Cadastre in Victoria Australia" Aien, Rajabifard, Kalantari, Williamson and Shojaei (2011) shows that work has been progressing steadily in this field for some time. Extended research for NSW hopes to learn from their experiences to date, and it is appreciated that the 3D model created here, while fit for purpose is not as advanced as that of other parties.

\section{BENEFITS OF CAPTURE}

Some of the benefits of LandXML capture as a complete dataset for the entirety of New South Wales are that it eliminates duplication and effort, is geospatial, and captures all plan information in a digital and versatile format. LandXML capture creates a numerical Cadastre that allows usage of observations and coordinates for a variety of stakeholders such as surveyors, GIS professionals, utilities, state and federal agencies, local government and internal Spatial Services business areas. Furthermore, stakeholders would benefit from the increased accuracy of the cadastre and its related datasets.

The key benefit of the back capture is that it eliminates duplication of effort. Until now every user of the plan data had to manually enter the plan information for use in their own geospatial or surveying and engineering environment. Being able to share one single source is extremely useful for geospatial work. A collection of LandXML files should provide a useful method of adjustment and correction for improving the accuracy of the DCDB. For new surveys it means that prior plan information and reference plans are ready for use and don't need to be captured again.

Strata schemes form part of this overall dataset. Currently strata properties are represented in the NSW DCDB by only what touches the surface. This means that any dwellings or other building structures existing above or below the earth's surface are not visible. While the DCDB's primary function has thus far been to represent the legal boundaries of land ownership on a 2D plane, it is clear that a dataset that caters for the location and extent of properties in 3D would be beneficial to its users and the community at large.

Such a dataset could have many potential uses for a number of agencies and stakeholders. Some of these may include emergency services, asset management, the property industry and various utilities. The future creation of a "proposed layer" showing developments that are to occur ahead of time could also be largely beneficial. A more localised benefit to Spatial Services and the primer for this work is to enable quality checking of Strata plans in LandXML. Staff will be able to check plans for errors and identify capture issues on a $3 \mathrm{D}$ model as opposed to a $2 \mathrm{D}$ set of points where all floors 
sit atop one another or, in the case of current practice, multiple sheets of paper.

\section{PROPOSED CHANGES TO LANDXML RECIPE AND VERSION}

The current version of the NSW LandXML recipe used is V8.0.2 (Land and Property Information, 2017). While changes to the version of the recipe in regards to strata capture are still in the discussion stages, the following are considered and were used as part of the trial capture:

The base plan was captured in a similar manner as per a regular Deposited Plan (DP):

$<$ Parcel name="55/1206312" class="Lot" state $=$ "affected" parcelType $=$ "Single" parcelFormat $=$ "Standard" buildingLevelNo="Location Plan" >

Strata lots were "nested" below this DP base parcel similar to the following:

$<$ Parcel name="Building 1" class $="$ Building" desc $=$ "Two Story Brick Units" state="proposed" parcelType="Single" parcelFormat="Strata" buildingNo="No99" buildingLevelNo="Location Plan"

Note the usage of the buildinglevelnumber attribute. This is captured as stated on the plan. However, for the purposes of creating a 3D model, an additional numerical attribute was added to the data that indicates floor number independent of this attribute. Levels could then easily be separated and extruded to any value.

- $\quad$-1 Lower Ground

- 0 Ground Floor

- 1 First floor

- 2 Second floor

- $\quad$...etc.

This is also useful as floor names as per plan could potentially be confusing for a future user of the file. What is labelled "first floor" on one plan may refer to a different physical level on a different plan.

Notes and designations such as the acronyms of parcel descriptions were recorded like the below:

$<$ Annotation type="Plan Note" name $=" 1 "$ desc $=$ "CYCourtyard"/>

$<$ Annotation type $=$ "Plan Note" name $=" 2 "$ desc="PPorch "/>
$<$ Annotation type $=$ "Plan Note" name $=" 3$ " desc="PKParking Space"/>

\section{CAPTURE OF PRE-EXISING STRATA PLANS IN LANDXML FORMAT}

The first step in creating the LandXML file was the capture of the original Deposited Plan that existed before the strata title was created. This is necessary as it is used as the "base plan" of which the internal buildings are to be related. Both the base DP and SP plan to be captured are required as images, in this case tiff format.

The base plan may already exist from prior work on the back capture program, in which case it can be copied and used as the basis for the SP. If the plan has not yet been captured, it needs to be using the standard capture procedure. A completed Strata LandXML will contain the base plan as well as the strata plan, as referenced in the previous section.

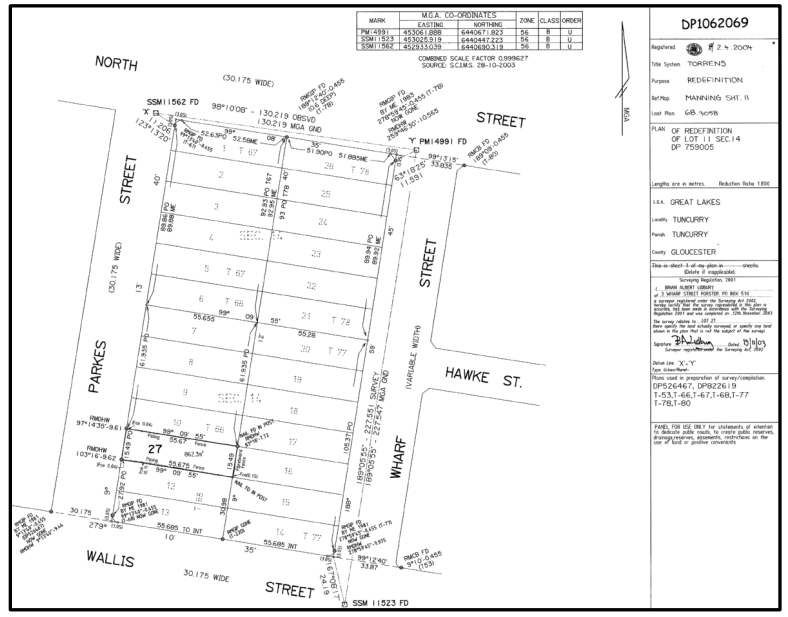

Figure 1: A deposited plan to be captured

For this study, the ArcGIS suite of tools has been used. To use the data in the LandXML file, the base plan was converted to a shapefile using an in-house developed tool and loaded into ArcMap. With this being displayed on screen, tiff images from the strata plan were able to be georeferenced, using the base plan extent to indicate the position of the internal buildings.

For ease of use, the tiff file was split into separate pages using a tiff editor, creating a separate image for each floor of the strata plan. The internal parcels for each floor were then created as polygons by tracing from the images, using the construction tools within ArcMap. The boundaries for parcels were created by tracing from the centre of the lot extents shown on the survey plan. This method did not take into account varying wall 
thicknesses; however this could be achieved by leaving a gap between each polygon rather than sharing common boundary lines. In current NSW strata schemes, ownership is based on the area measured to the shared walls surface, not its centre. Appropriate attribution for each polygon was entered as per the proposed LandXML recipe version.

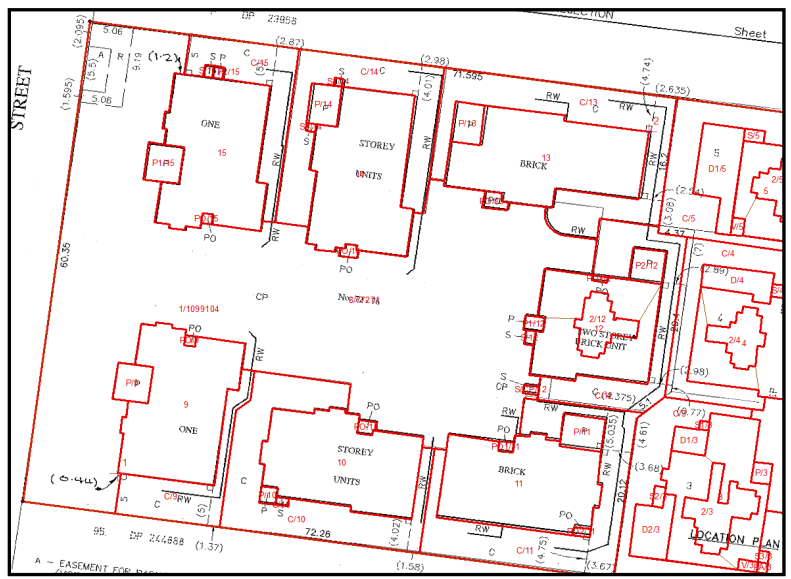

Figure 2: Tracing internal parcels of a georeferenced image

Strata plans in NSW contain offsets, that link and give measurement from the internal lots to the boundary of the base plan. These offsets were captured as connection lines, ensuring that internal lots are connected to the boundary and are not left "floating" and unconnected. Connections to survey control were also captured where possible so that the plan may be spatially enabled with other projected datasets.

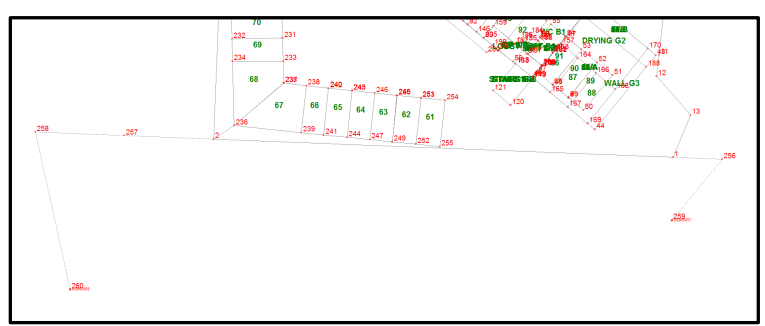

Figure 3: Capture of survey control and connection lines

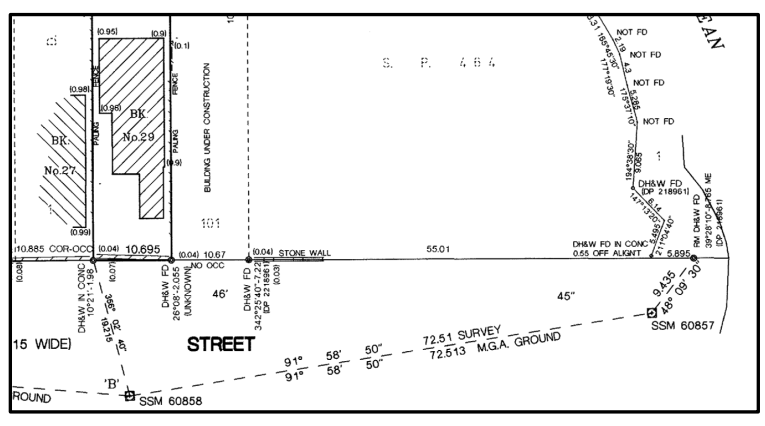

Figure 4: A plan showing survey control lines

Each floor plan in the strata entitlement was captured using the same method. NSW Strata plans are often not fully dimensioned, so the tracing method and subsequent creation of polygon parcels leads to approximated bearings and distances only. After all pages of the plan have been captured as specified, the shapefile is exported to GeoCadastre (Geodata Australia), which can be used to add dimensions and any other required attribution to the completed LandXML file. Following this process, differences can be checked after capture by calculating the area of the polygons as compared to the stated areas on the plan. In the test plans so far, the areas tended to be around $+/-5 \%$ difference.

After completion, the file may once again be converted to a shapefile for use with ArcGIS tools. This process was performed to enable a suitable method to examine the plan in a three dimensional space.

\section{CREATION OF A 3D MODEL}

Part of the Spatial Services back capture project is checking and quality assurance of captured plans. For current two dimensional captures, it is easy to visualise the plan to complete this process. A multi levelled strata plan however is much more difficult to view on a $2 \mathrm{D}$ plane. As such, a basic method that can quickly show a plan in $3 \mathrm{D}$ is required for our quality checking purposes.

It should be noted that the work performed here is not truly the creation of a full 3D model. Rather, the two dimensional capture of the LandXML file detailed above will be extruded to create the illusion of a full three dimensional capture. The following diagram illustrates the differences in these two approaches. 


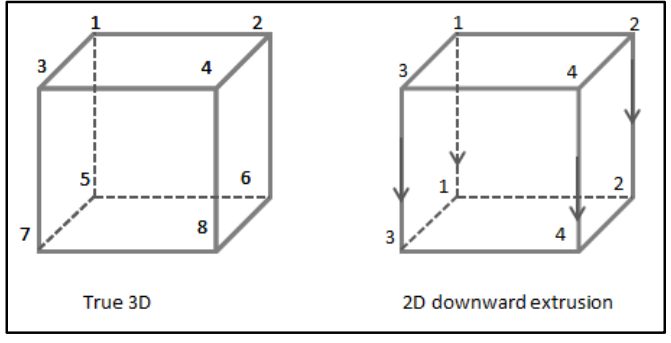

Figure 5: True 3D versus 2D extrusion

One downfall to this approach is that curved or slanted surfaces between floors cannot be accurately represented. Rather, they are merely extruded as blocks with flat faces for the chosen value until they change at the surface of the floor below or above.

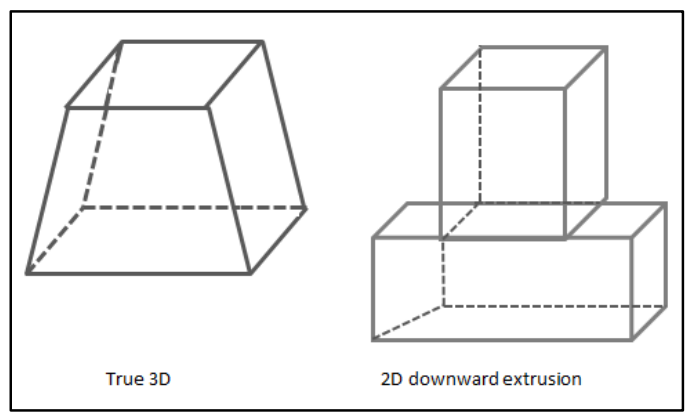

Figure 6: A shape that cannot be represented by 2D extrusion

The benefits of 2D extrusion over full 3D capture are that it is quick and relatively easy to get a basic model suitable for a variety of purposes such as quality assurance, uses less processing power, and does not rely on advanced 3D modelling software. For this study, ArcScene was used as the tool to represent the captured strata plan as a model. This program forms part of the ArcGIS environment currently used by Spatial Services.

The BuildingLevel attribute captured earlier was used as the basis for extruding the model. While a unit of 'one' was captured to indicate the difference between each floor plan, this value can be extruded through ArcScene to any value to simulate real world heights more accurately. Three metres was chosen as a basic approximation for the height of each floor in the example.
For more impressive visualisation, the final model can be improved with the addition of flat imagery, surface models, or a combination of any other geospatial content.

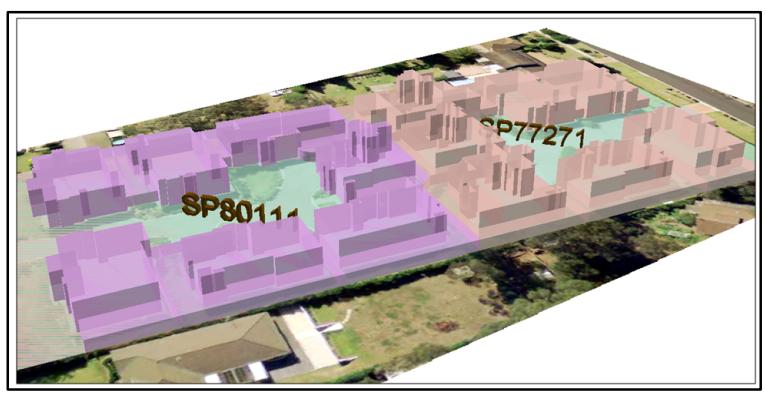

Figure 7: A staged strata development on a flat image

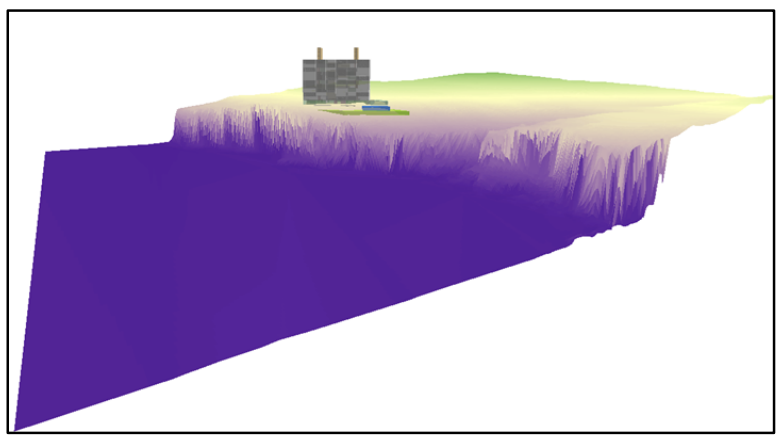

Figure 8: A strata plan on top of a surface model

The finished result pictured below shows a small patch of high quality $10 \mathrm{~cm}$ imagery covering a larger patch of lower quality imagery, chosen to improve processing speed. The imagery has been draped over a surface model, which increases the realism of the hill and cliff face, and gives a good indication of the real world lay of the land. For quality checking of a plan none of this is required, however it gives some insight as to how a quick and easy "skeleton" model could be used as an anchor for other datasets.

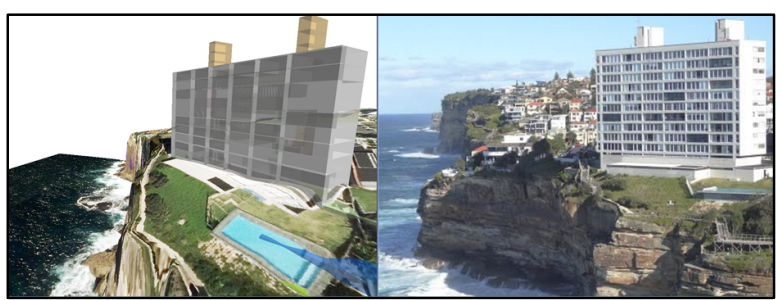

Figure 9: Captured model vs real life. Image on right "Diamond Bay - Ugly Building" @) Google Maps 2013 


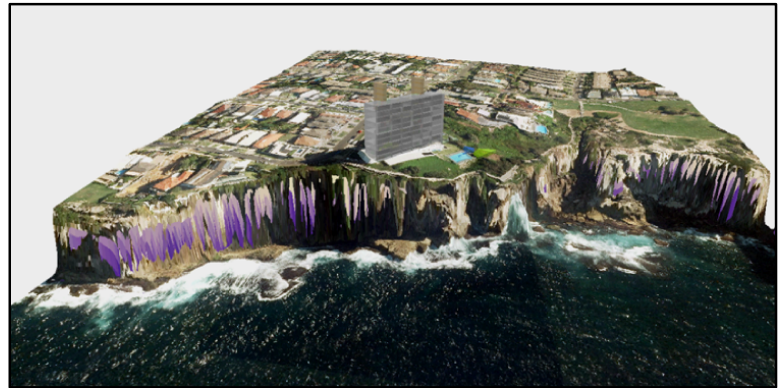

Figure 10: Captured strata plan on surface model with draped imagery

\section{ISSUES AND SCENARIOS ENCOUNTERED}

Some of the more interesting scenarios encountered during the test captures were:

7.1 Difficulty in determining locations of some parcels. The below example shows where location had to be approximated from comparison to aerial imagery, as there is no other indication on the plan of where the parcels sit.

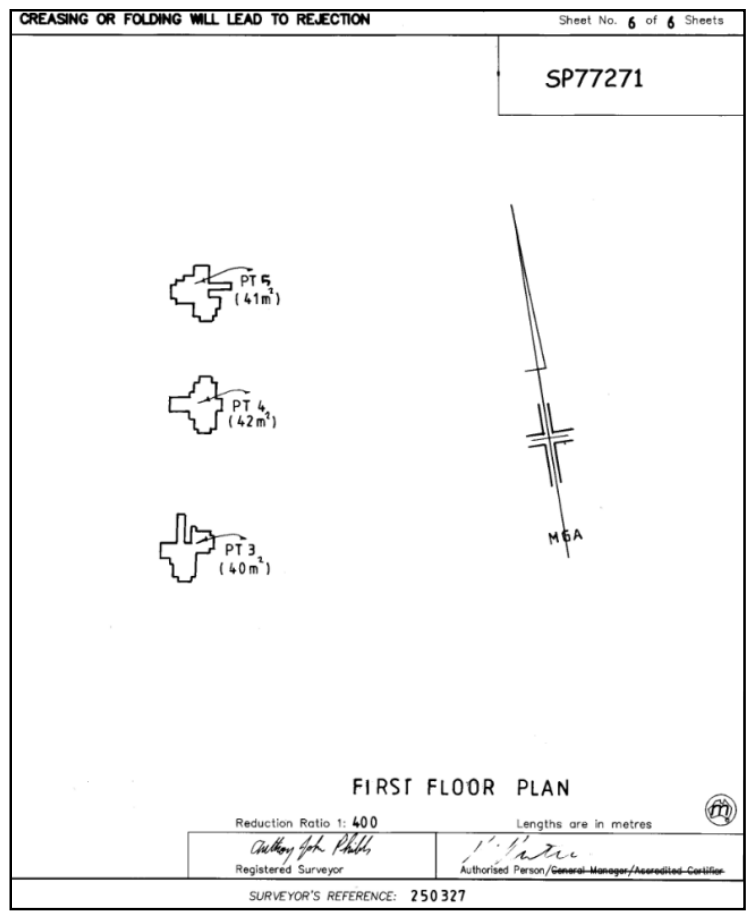

Figure 11: Floor plan not indicating location

7.2 Acronyms and other administration data are not standardised across different plans. Care needs to be taken to ensure that these are mapped correctly in the final LandXML.

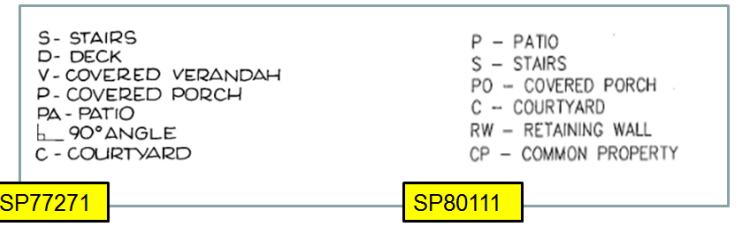

Figure 12: Acronym difference across different plans

7.3 In the creation of the 3D model, some plans have duplicated floor plans over multiple levels. This allows parcels to be copied and pasted for each floor rather than being traced individually so long as their attribution was correctly updated.

7.4 While only strata properties have been captured so far, it is envisaged that stratum could be captured in a similar manner. Many of these are not currently recorded in the DCDB, as they sit above or below the earth's surface.

\section{LIMITATIONS OF THE APPROACH TAKEN}

It is appreciated that the method outlined in this paper is not a perfect one. Rather, it is a relatively quick and easy way to represent strata plans in a three dimensional environment. It fits the purpose of being able to quality check LandXML captured plans. As dimensions are not required to be recorded on strata plans registered in NSW, the tracing method leads to parcels having only approximated bearings and distances. In the longer term it needs to be decided if these approximations (around +/$5 \%$ difference to stated area on plan) are accurate enough for the usage within the DCDB.

Similarly, NSW strata plans are not required to show heights or cross sections between floors. Plans that do not contain this data will not be as accurately represented where they are sloped, or there is some other change between the floors. Furthermore, changes between levels cannot be shown accurately using the $2 \mathrm{D}$ extrusion method shown here.

As also experienced by Shojaei, Olfat,Rajabifard, Darvill and Briffa (2016), curved surfaces are difficult to produce while tracing parcels. Rather, they are approximations made up of short small lines that become flat faced segments when projected, and are dependent 
on how particular the capturing officer has been while tracing.

\section{CONCLUSION AND FUTURE INVESTIGATIONS}

Through the short trial study conducted, it is clear that Strata plan capture in LandXML is necessary to attain a complete back captured LandXML dataset of plans registered in NSW. This data could be valuable for a variety of stakeholders.

It is also clear that to be able to fully realise the captured LandXML, projection into some sort of 3D model is of value, especially for enabling the quality checking of plans.

More fully realised software could be beneficial for ease of modelling. In their research article "Building Information Modelling for High-rise Land Administration, Atazadeh, Kalantari, Rajabifard, Ho and Ngo (2016) suggest that BIM could be used for this purpose, with some modifications to allow for proper recording of land administration attribution. The authors support further investigation into this approach.

Future integration between datasets may utilise 3D image capture technology to give the plan a realistic facade. For example, imagery of a building could be photographed by a drone and draped over the strata model. This would allow for both internal and external representation within a 3D environment. Other datasets such as addressing could also be integrated within the same 3D space and be of high value to emergency and utility services.

\section{ACKNOWLEDGEMENTS}

The authors would like to thank the ICSM ePlan Working Group, NSW ePlan team and Spatial Services staff. This paper is based on the view of the authors and may not represent the view of DFSI Spatial Services.

\section{REFERENCES}

Aien, A., Rajabifard, A., Kalantari, M., Williamson, I., and Shojaei, D., 2011. "3D Cadastre in Victoria Australia - Converting Building Plans of Subdivision", University of Melbourne, Australia.

https://www.gim-international.com/content/article/3dcadastre-in-victoria-australia (10th April 2017)
Atazadeh, B., Kalantari, M., Rajabifard, A., Ho, S., and Ngo, T., 2016. "Building Information Modelling for High-rise Land Administration”, University of Melbourne, Australia https://www.researchgate.net/publication/293325900 Bu ilding Information Modelling for Highrise Land Administration (7th April 2017).

Hornery, A., 2015 "Single Land Cadastre for NSW Summary of Outcomes", Sydney, Australia. http://spatialservices.finance.nsw.gov.au/ data/assets/pd f_file/0019/205642/Single_Land_Cadastre_NSW__Summary_Outcomes_Report.pdf (11th April 2017)

Intergovernmental Committee on Surveying and Mapping (ICSM), 2015. "Cadastre 2034 - Powering Land \& Real Property" Canberra, Australia http://www.icsm.gov.au/cadastral/Cadastre2034.pdf (6th April, 2017)

Land and Property Information, 2017. "NSW LandXML Recipe -

Specifications for preparation of Deposited Plans in LandXML format for lodgment in LPI NSW" Sydney, Australia.

http://www.lpi.nsw.gov.au/_data/assets/pdf_file/0019/1 38034/NSW LandXML Recipe 8.0.2.pdf (6th April 2017)

Shojaei, D., Olfat, H., Rajabifard, A., Darvill, A., and Briffa, M. 2016. "Assessment of the Australian digital cadastre protocol (ePlan) in terms of supporting 3D building subdivisions", Land Use Policy Journal, Volume 56, November 2016, Pages 112-124. http://www.sciencedirect.com/science/article/pii/S02648 37715300818 (7th April 2017) 